\title{
Autonomous vehicles for remote sensing use - Opportunities and challenges for Drone
}

\author{
Jean-Louis LAURONT ${ }^{1 *}$, Michel GUILHOT ${ }^{2}$, Pascal HARNOIS ${ }^{3}$ and Gary STRAHAN ${ }^{4}$ \\ ${ }^{1}$ INNOVIDEA SAS, President, 91370 Verrieres le Buisson, France \\ ${ }^{2}$ CDSI, Technical Director, 75008 Paris, France \\ ${ }^{3}$ HAZIEL, President, 95000 Cergy, France \\ ${ }^{4}$ INFRARED CAMERAS IncI, Chief Executive Officer, Beaumont, TX 77705 USA
}

\begin{abstract}
Emergence of autonomous robotics and vehicles thanks to sensors, embedded processors and communication progress open new horizon for making remote sensing measurement without exposing human to unecessary risks. The collection of regular data in various environement (outdoor or indoor) allow more efficient predictive and preventive maintenance of assets that are difficult to access. Autonomous vehicle operating on ground, in the air or on water can shape a new future for science, industry and environnement. The optical and photonic industries offer now a wide range of compact sensor solutions using different wavelength not only to permit autoguidance of vehicles but also to complete the range of sensor already used for gathering various physical values with the advantage of non-contact. The application are numerous and encompass amongst other thermography, gas detection, hyperspectral imaging, security which can be extremely helpful for carrying day to day tasks for first responder, EHS officers, governmental regulatory organization, scientists... Routine checks can be thus envisaged that will save money by preventing or lessening risks or failure, better protecting environment and people, capitalizing on larger databases making use of AI. INNOVIDEA through its diverse industrial innovative partners will illustrate several example of use with drone (UAV/UAS/RPAS)
\end{abstract}

\section{Autonomous vehicles}

\subsection{Introduction}

Generally speaking a majority of people think about passenger cars, buses or trucks when they hear about the term « autonomous vehicle » but the potential or already existing markets for autonomous vehicles actually represent a much larger domain. Different vehicle type can indeed be considered regarding the media and the number of dimension there are evolving in or into; 2D (ground, above water/surface), 3D (air, underwater, underground). Potential use of those autonomous vehicles are beyond imagination when someone think about the markets ; transport, industry, agriculture and environment, defence and security, space exploration...

\subsection{Full autonomous navigation}

Autonomous vehicle possess different level of autonomy going from partially remotely controlled to full autonomy operation for both their navigation and mission. Vehicle design and operation complexity are both directly linked to the aimed autonomy of navigation level. Numerous sensors (such as LIDAR, radar, CMOS imager or time imager, ultrasonic sensor, IR sensor, GPS, RTK, IMU), on-board processing (hardware, firmware, software), power supply and communication systems are a necessary pre-requisite for the reliable operation before considering embarking remote sensing instruments for making measurements.

The highest level of navigation autonomy encompasses « sense and avoid » that must be considered in a global manner taking into account environment (incl. weather, constraints), potential failure scenarios and adapted safety margin (FMEA). Therefore, multi sensor approach and comunnicatiion means with a certain level of redundancy and complex preprogrammation of embedded/remote processing is required. Range of measure as well as field of « view » coverage must be adapted to vehicle speed and ability of this one to react on time by changing trajectory, breaking, landing for instance in order to avoid collision or to be stuck in a position it cannot escape.

* Corresponding author info@,innovideasas.com 


\section{Drone (Unmanned Aerial vehicle)}

\subsection{Operating modes and mission}

The rapid expansion of drone offer great opportunities for using this aerial vehicle which can be monitored in different mode (remotely piloted or on preprogrammed 3D enveloppe or in complete/full navigationautonomy) for remote sensing use.

The most common useage in the measurement domain at this point are LIDAR (3D point cloud scanning), photogrammetry, thermography, magnetometry but new areas such as gas detection (sniffer, optical gas imaging, spectrometer), Corona, Air sampling, ultrasonic measurement are promising.

Moreover, the use of drone to install remote sensor in difficult to access location or to read regularly via RFID for instance pre-installed sensor also in remote location is only at the verge of development. Not to mention another area for drone that consists in acting ; spraying or injection of liquids, robotic arms,...

\subsection{Semi-autonomous navigation and related payloads application}

\subsubsection{Mixed mode example}

DJI drone maker proposed to user for some of their mission, the possibility to define through its man to machine interface a preset enveloppe of flight setting therefore the limit of flight. Moreover several elaborated preprogrammed function called RTH (return to home) functions for safety reasons are implemented inside operating software.

The RTH function has three type mode : Low Battery RTH (power), Failsafe RTH (communication), smart RTH

Low Battery RTH ; an «Intelligent Flight Battery » algoritm manage remaining available power. Depending on battery level, drone comes automatically to a RTH height and back to its taking off location or land immediatly (if critical low level is reach). In this last case, of course, consequences may be serious including crash or collision hence the importance of battery management or surveillance by pilot.

Failsafe RTH : in case of loss of signal for a given duration depending on communication mean (wifi or radio) several scenarii will take place be proposed or imposed to pilot ; hover,

\subsubsection{Remote sensing payload for building}

Haziel in France who uses DJI drone equipped with high resolution CMOS camera (photogrammetry) or LIDAR is achieving Building Information Modelling (BIM) in outdoor or indoor for architectes, heritage conservation assessments.

Here are illustration of two type of remote sensing done with this type of light aerial vehicles. It is to be noticed the high quality of rendering (texture, color) with photogrammetry (Fig .1.) and the high accuracy of dimensional measurement in the $0,5 \mathrm{~mm}$ range at $60 \mathrm{~m}$ distance for LIDAR (Fig.2. \& 3.). 


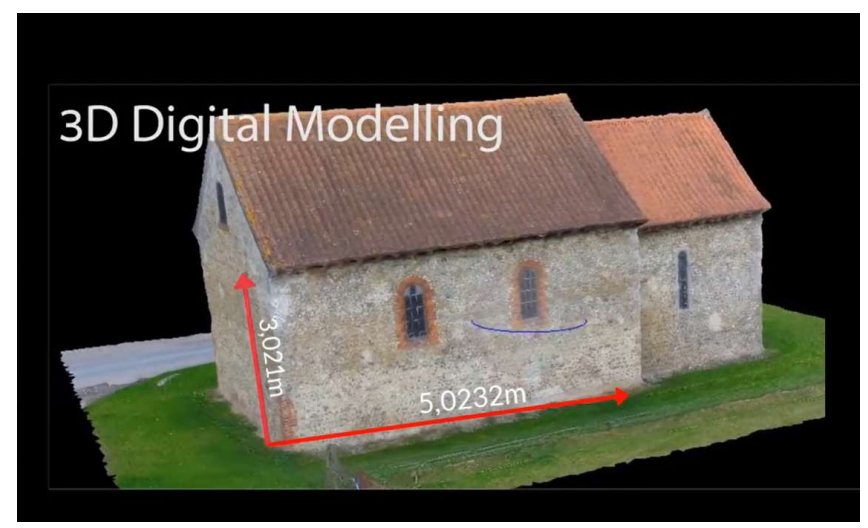

Fig.1. Assembled file of church done with photogrammetry

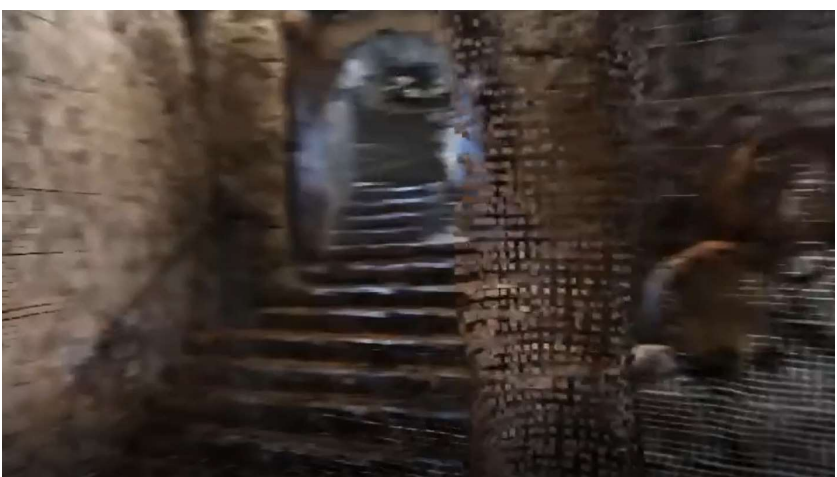

Fig.2. Point cloud capture by LIDAR inside castle

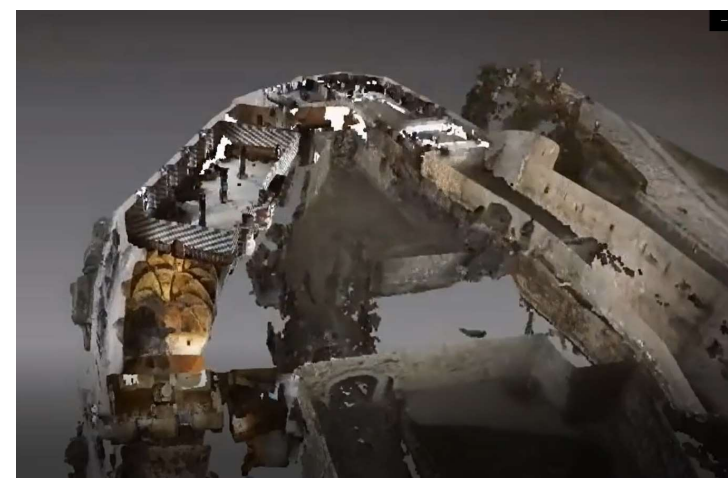

Fig.3. Digital reconstruction of entire castle

\subsubsection{Remote sensing payload for gas leak detection}

Infrared Cameras in USA has developped a turn key solution for thermography and gas detection also called Leak detection and repair LDAR (by Optical Gas Imaging OGI and TDLAS spectrometer) that can be mounted on drone compatible with A3 and Lightbridge 2 DJI interface.

Main performances of instruments for hydrocarbon gases and VOC leaks detection, quantification and localization are as follows :

- $14 \mathrm{mK}$ NETD at $30^{\circ} \mathrm{C} \mathrm{f} / 1.0$ and a $+/-1 \%$ accuracy for the cooled MWIR (3-5microns) OGI camera that offers $0.75 \mathrm{~g} / \mathrm{h}$ methane treshold,

- 0.5 to $50 \mathrm{~m}$ range detection ( 30 to $120 \mathrm{~m}$ for longer range), $1 \mathrm{ppmxm}$ treshold for methane and 1 to 50000 ppm range, response time 0.5 second.

The complete architecture for their turnkey complete gas leak detection and quantification payload is illustrated in diagram beneath.(Fig.4.) 


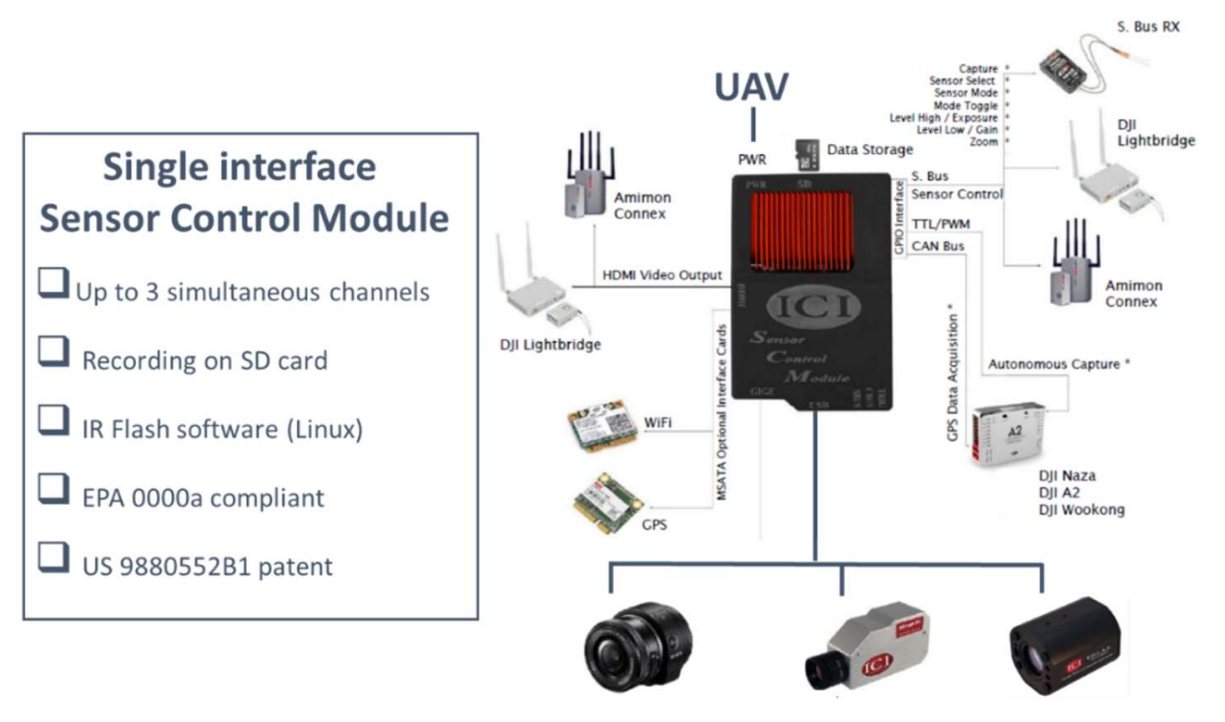

Fig.4. Optical Gas Imaging and TDLAS payload and interface architecture incl. Sensor Control Module (ICI)

Their main innovation consists in the fact that then can remote controlled up to three measurement channels simultaneously in real time from the ground. This is achieve through a Linux program through the DJI app interface. TDLAS spectrometer, OGI camera work in cooperation complemented by high resolution visible CMOS camera.

The live video from both visible and infrared camera where measurement value of TDLAS is displayed (axis of TDLAS is materialized inthe center of infrared image with an incrusted tiny circle) can be visualized by an operator. As soon as detection of gas leak by TDLAS (typical concentration value $>$ two digits number ppm) or by camera** (gas turbulence in the image like fume or air distorsion aspect) or by both occurs the operator will then move drone so that the instruments show on the image maximum value of TDLAS displayed. This technique allows to rapidly narrow down the uncertainty on gas leak exact localization. As soon as this achieved and while drone is maintained in strict fixed position, an high definition still image is recorded as well as a 3 seconds movie from OGI camera where methane concentration level is incrusted. Unlike other existing technologies, operator can have an immediate access to datas from the three combined optical instruments.An example report is shown hereafter.

** To be noted that operator can change mode of OGI camera (color palette, hot white/black, differential video mode) facilitating thus the visualization of gas leaking expecialluy if leak is small. 
Inspection Report

\begin{tabular}{|c|c|c|c|}
\hline Project: & Session: Octob & $15,201821.18 .18$ & Image: 0018_MWIR \\
\hline Project & & Date & $2018 / 10 / 15$ \\
\hline Company & E & Time & $21: 38: 032$ \\
\hline Contact & & Location & Is Flare \\
\hline Address 1 & L & Ambient & $11 \mathrm{C}$ \\
\hline Address 2 & $=260$ & Distance & $25 \mathrm{~m}$ \\
\hline City & & Methane Sensor Data & 4745 ppm_m \\
\hline State & & GPS Lattude & \\
\hline Zip & & GPS Longitude & \\
\hline Phone & & GPS Altude & 687.866760 \\
\hline Email & h.com & GPS Heading & 38.176540 \\
\hline
\end{tabular}
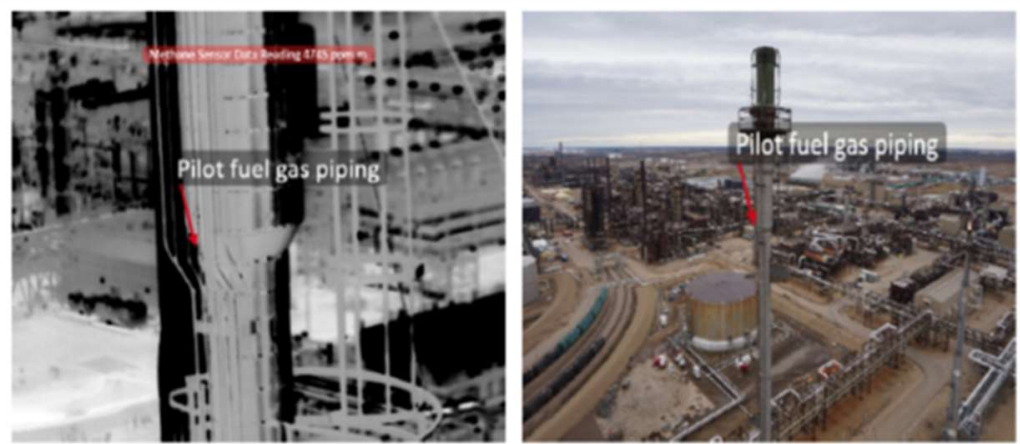

Annotation: Pilot fuel gas piping

\begin{tabular}{|l|l|}
\hline Comment & $\begin{array}{l}\text { During an Unmanned Aerial Vehicle flare stack inspection, technicians utilizing an ICI Optical Gas } \\
\text { Imaging (OGI) camera and Methane Laser Spectrometer (MLS) found a leak on Acid Gas Flare } \\
\text { pas supply welded piping at a height of approximately } 60 \text { meters above ground. }\end{array}$ \\
\hline
\end{tabular}

Fig.5. Sample report with still high resolution visible image and OGI 3 seconds video with TDLAS measurement value (ICI)

In future, automatic algorithm can be imagined to replace the operator action and run fully automatic inspection. Two challenges still remain to be solved power autonomy of drone typically for pipeline and reliable communication system.

\subsection{Full autonomous navigation}

\subsubsection{An innovative contrarotative drone}

The DGAC homologated FS-S3 drone (Fig.6.) from CDSI is based upon a patented propulsion principle with contra-rotative propellers powered by electrical motor and u-shape battery. It is equipped with protection allowing it to work « in contact » which is of particular benefit for bridge, chemney, buildings inspection (Fig.7.) Its aeronautical characteristics ensure a high stability of flight even in strong gusting winds. The standard operation mode use a proprietary secured wireless communication with triple redundancy.

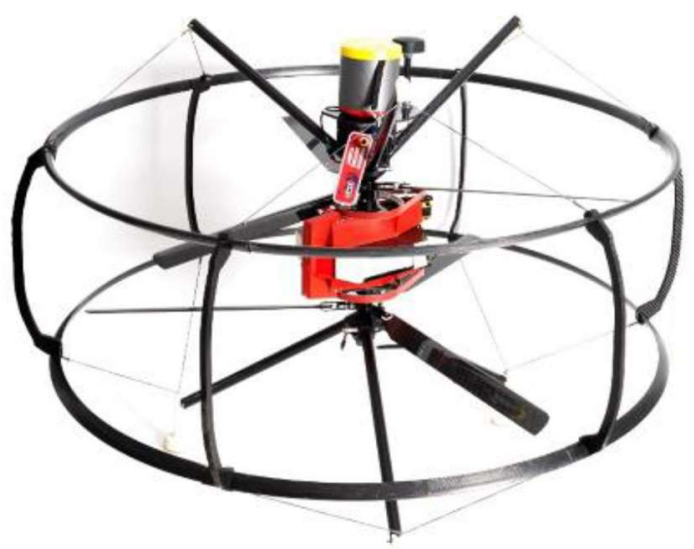

Fig.6. FS-S3 Drone (CDSI) 


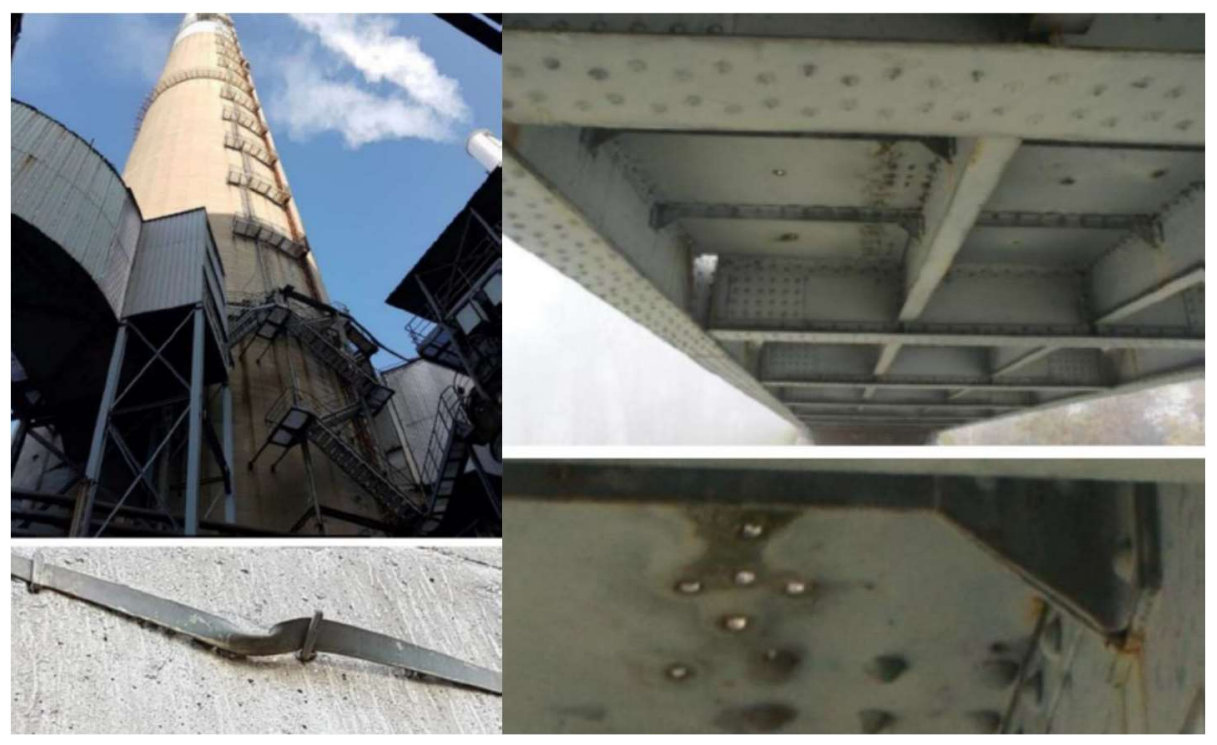

Fig.7. Left - 240m chemney inspection (lightning ground lead), Right-bridge rivets inspection

\subsubsection{Example of full autonomous navigation with FS-S3}

This aerial vehicle can also operate in fully automatic mode using waypoint ; this has been used with FS-S3 with for nuclear environement in order to collect air sampling and to drop radiation probe on the ground (Fig.8. and Fig.9.).

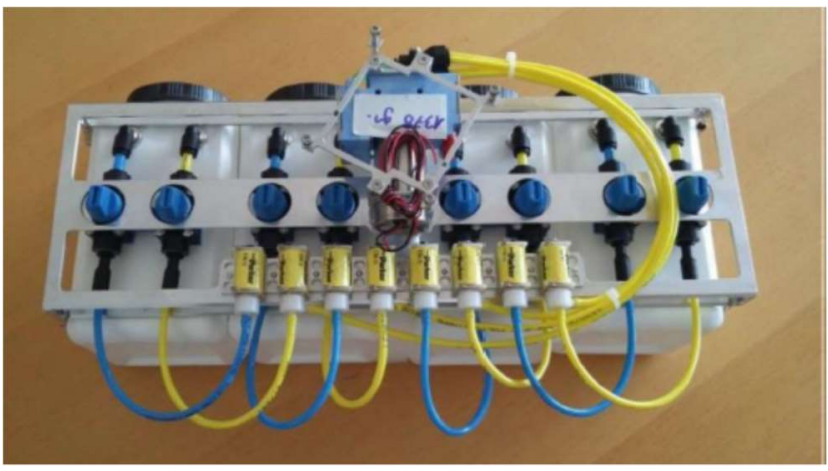

Fig.8. Left - Atmospheric sampling Unit (4)

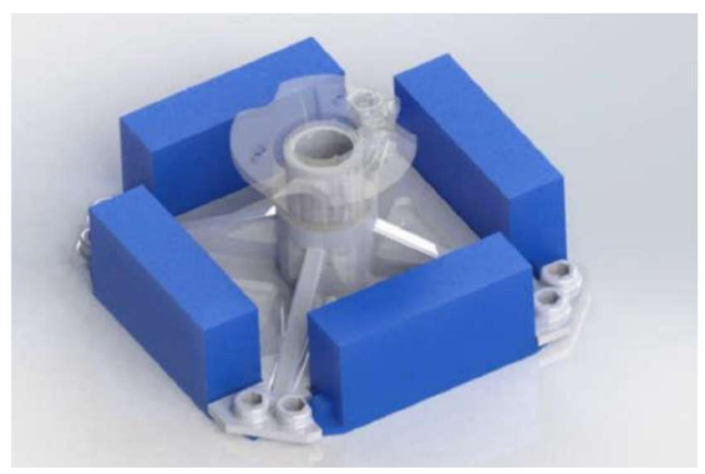

Fig.9. Radiation probe larging unit

Principle of autonomous navigation :

\section{Initial Settings}

a) a satellite map of the site to inspect is downloaded into the Man to Machine Interface,

b) user then mark on the resistive tactile screen the different exact point coordinates $(\mathrm{X}, \mathrm{Y})$ called « waypoint » on map where drone will make a specific action

c) the height is predefined hence completing the exact 3 localisation.

Flight sequence

a) Drone can now start in autonomous navigation flight mode and take off.. The drone localisation in 3D is ensured by GPS ( $\sim 60 \mathrm{~cm}$ precision in two axis X,Y) and RTK (centrimetric precision) and altimeter sensor. Its localization, speed and other key parameters are transmitted via the triple redundancy communication system

b) It will reach its first waypoint using preprogrammed slope to arrive at the required height and with variable flight speed (high then low when approaching the desired waypoint). Uncertainty of localisation is taking care of by RTK. The drone will not be able to move towards next waypoint unless it has passed at the previous waypoint. Moreover the software handled the wind condition in order to 
ensure actual reach of waypoint thorugh command of with propeller angle (close loop system). Unlike other multirotor that need to increase rotor speed which result in using mucgh faster battery power, FS$\mathrm{S} 3$ has constant rotation speed and therefore does not consume extra power.

c) Once waypoint reached, the program activates the electromagnet valves for collectiing air sample or for dropping probe depeding on selected payload. Then the drone continues its fligth to the next waypoint.

\section{Conclusion}

The future of autonomous vehicle for remote sensing use -partially illustrated here for drone (limited here by the way to multicopters examples) - will likely rapidly grow thanks to the cost benefits it may bring for difficult to access sites. The complexity of sensors, embedded computing/firmware/software programming and communication with remote station for allowing full autonomous operation has been evoked. Design and reliability of such systems remain a considerable challenge when taking into account the numerous environment parameters like communication availability and consitency, weather (wind, temperature, visibility) or other various factors (regulation, dust, animals,...)

As well, the remote sensing payloads should be considered as full part of system when it comes to their characteristics of weight, size, power consumption, interface and environmental performances. The source and amount of energy to power drone is the number one challenge as its autonomy is directly limited by this factor. The field and reach of use for precise measurement instruments embarked on aerial vehicles is just at the beginning era. Their flight automation would open huge markets wherever large infrastruture or territory must be cover such as power gird, oil or gas pipeline, railways or in a different market segment for confined environment with poor or no communication means (mines, tunnel, pipes,...).

Finally, the autonomous aerial vehicles used in outdoor are fully complementing the space observation networks already in place or to come. Core market is the data and a fast coming trend will be to store these datas directly on the cloud hence benfiting from latest $\mathrm{AI}$ and deeplearning progress ; this will be reinforced by $5 \mathrm{~g}$ deployment but this is another theme.

\section{Abreviation}

AI : Artificial Intelligence

BIM: Building Inspection Modelling

CMOS: Complementary Metal Oxide Semi-conductor

DGAC: Direction Générale de l'Aviation Civile

EHS : Environment Health and Safety

FMEA : Failure Mode and Effect Analysis

IMU : Inertial Measurement Unit

LDAR : Lead Detection And Repair

LIDAR: LIght Detection And Ranging

OGI: Optical gas Imaging

RFID: Radio Frequency IDentification

RPAS: Remotely Piloted Aerial System

RTK: Real Time Kinematic

TDLAS: Tuneable Diode Laser Absoprtion Spectrometer 
UAS: Unmanned Aerial System

UAV: Unmanned Aerial Vehicle 